\title{
Understanding hand hygiene behaviour in the intensive care unit to inform interventions: an interview study
}

Kathryn Lambe ${ }^{1,2}$, Sinéad Lydon²,3, Caoimhe Madden ${ }^{1,2}$, Jenny McSharry ${ }^{4}$, Rebecca Marshall5, Ruth Boylan ${ }^{6,7}$, Aoife Hehir ${ }^{3}$, Molly Byrne ${ }^{4}$, Omar Tujjar ${ }^{7}$ and Paul O'Connor ${ }^{1,2^{*}}$

\begin{abstract}
Background: Improving hand hygiene $(\mathrm{HH})$ compliance is one of the most important, but elusive, goals of infection control. The purpose of this study was to use the capability $(\mathrm{C})$, opportunity $(\mathrm{O})$, motivation $(\mathrm{M})$, and behaviour (B; COM-B) model and the theoretical domains framework (TDF) to gain an understanding of the barriers and enablers of $\mathrm{HH}$ behaviours in an intensive care unit (ICU) in order to identify specific interventions to improve HH compliance.
\end{abstract}

Methods: A semi-structured interview schedule was developed based upon the COM-B model. This schedule was used to interview a total of 26 ICU staff: 12 ICU nurses, 11 anaesthetic specialist registrars, and three anaesthetic senior house officers.

Results: Participants were confident in their capabilities to carry out appropriate HH behaviours. The vast majority of participants reported having the necessary knowledge and skills, and believed they were capable of carrying out appropriate HH behaviours. Social influence was regarded as being important in encouraging HH compliance by the interviewees- particularly by nurses. The participants were motivated to carry out $\mathrm{HH}$ behaviours, and it was recognised that $\mathrm{HH}$ was an important part of their job and is important in preventing infection. It is recommended that staff are provided with targeted $\mathrm{HH}$ training, in which individuals receive direct and individualised feedback on actual performance and are provided guidance on how to address deficiencies in $\mathrm{HH}$ compliance at the bedside at the time at which the HH behaviour is performed. Modelling of appropriate $\mathrm{HH}$ behaviours by senior leaders is also suggested, particularly by senior doctors. Finally, appropriate levels of staffing are a factor that must be considered if $\mathrm{HH}$ compliance is to be improved.

Conclusions: This study has demonstrated that short interviews with ICU staff, founded on appropriate behavioural change frameworks, can provide an understanding of $\mathrm{HH}$ behaviour. This understanding can then be applied to design interventions appropriately tailored to the needs of a specific unit, which will have an increased likelihood of improving $\mathrm{HH}$ compliance.

Keywords: Hand hygiene, Infection control, Compliance, Intensive care, Interview, Behaviour

\footnotetext{
* Correspondence: paul.oconnor@nuigalway.ie

'Discipline of General Practice, School of Medicine, National University of Ireland Galway, Co. Galway, Ireland

Irish Centre for Applied Patient Safety and Simulation, School of Medicine, National University of Ireland Galway, Co. Galway, Ireland

Full list of author information is available at the end of the article
}

(C) The Author(s). 2020 Open Access This article is licensed under a Creative Commons Attribution 4.0 International License, which permits use, sharing, adaptation, distribution and reproduction in any medium or format, as long as you give appropriate credit to the original author(s) and the source, provide a link to the Creative Commons licence, and indicate if changes were made. The images or other third party material in this article are included in the article's Creative Commons. licence, unless indicated otherwise in a credit line to the material. If material is not included in the article's Creative Commons licence and your intended use is not permitted by statutory regulation or exceeds the permitted use, you will need to obtain permission directly from the copyright holder. To view a copy of this licence, visit http://creativecommons.org/licenses/by/4.0/ The Creative Commons Public Domain Dedication waiver (http://creativecommons.org/publicdomain/zero/1.0/) applies to the data made available in this article, unless otherwise stated in a credit line to the data. 


\section{Background}

Improving hand hygiene $(\mathrm{HH})$ compliance is one of the most important, yet elusive, goals of infection control [1]. Although the $\mathrm{HH}$ procedure itself is simple, the behaviours related to consistent engagement with $\mathrm{HH}$ are complex and not readily understood, explained, or changed $[2,3]$. A commonly used approach to improve compliance is to use a bundled $\mathrm{HH}$ intervention whereby several strategies are used together [2, 4]. However, this method may not constitute the most effective use of limited resources and there is a need to consider all interventional strategies employed, their necessity and sufficiency $[2,4,5]$. It has been suggested that the development and/or the selection of interventions to implement changes in healthcare practice is often conducted on the basis of intuition [6-8], and, correspondingly, very few studies of interventions to improve $\mathrm{HH}$ compliance are grounded in behaviour change theory [9]. Therefore, to best identify how to improve $\mathrm{HH}$ compliance, we first require an understanding of the determinants of current and desired behaviours informed by a theory of behavioural change.

There are many different theories of behaviour change, often with similar and overlapping constructs [10]. The diversity and number of these theories have been identified as possible reasons why theoretical approaches are not used in the design of interventions [11]. In response to these issues, the Theoretical Domains Framework (TDF) was developed in order to provide a structure to support the application of theoretical approaches to interventions with the goal of fostering behavioural change $[12,13]$. The framework supports an evidence-based approach to the identification of interventions that are most likely to bring about changes in behaviour. The TDF consists of 14 domains. It was designed using a systematic consensus approach to simplify and amalgamate the numerous behavioural change theories relevant to behavioural change [13]. The TDF has been used as a framework to study barriers to $\mathrm{HH}$ in the past [3, 14-17].

A related model is the COM-B model, in which three factors interact to generate behaviour: the individual's capability $(\mathrm{C})$, opportunity $(\mathrm{O})$, and motivation $(\mathrm{M})$ to engage in a given behaviour (B). The COM-B model is a compilation of 19 different behavioural change frameworks and complements the TDF as a tool for understanding behaviour and supporting development of appropriate interventions [11]. The benefit of the COM-B model over other, more established theories is that it is designed to be simple yet comprehensive and describes the minimum number of factors needed to account for behaviour change. As can be seen from Table 1, each of the COM-B domains maps onto several unique TDF domains.

The purpose of the study described in this paper was to use the COM-B model and the TDF as an analytical framework to gain an understanding of the barriers and enablers of $\mathrm{HH}$ behaviours in an intensive care unit (ICU) setting through semi-structured interviews with ICU healthcare providers. Qualitative methods support a

Table 1 Theoretical domains framework and COM-B domains (adapted from Michie et al., 2014 [10])

\begin{tabular}{|c|c|c|}
\hline COM-B & TDF Domain & Definition \\
\hline \multirow[t]{5}{*}{ Capability } & Knowledge & An awareness of the existence of something. \\
\hline & Skills & An ability or proficiency acquired through practice. \\
\hline & Beliefs about capabilities & Acceptance of the truth, reality, or validity about outcomes of a behaviour in a given situation. \\
\hline & Behavioural regulation & Anything aimed at managing or changing objectively observed or measured actions. \\
\hline & $\begin{array}{l}\text { Memory, attention, \& } \\
\text { decision processes }\end{array}$ & $\begin{array}{l}\text { The ability to retain information, focus selectively on aspects of the environment and choose between } \\
\text { two or more alternatives. }\end{array}$ \\
\hline \multirow[t]{2}{*}{ Opportunity } & Social influences & $\begin{array}{l}\text { Those interpersonal processes that can cause individuals to change their thoughts, feelings, or } \\
\text { behaviours. }\end{array}$ \\
\hline & $\begin{array}{l}\text { Environmental context and } \\
\text { resources }\end{array}$ & $\begin{array}{l}\text { Any circumstance of a person's situation or environment that discourages or encourages the } \\
\text { development of skills and abilities, independence, social competence and adaptive behaviour. }\end{array}$ \\
\hline \multirow[t]{7}{*}{ Motivation } & $\begin{array}{l}\text { Social/professional role and } \\
\text { identity }\end{array}$ & $\begin{array}{l}\text { A coherent set of behaviours and displayed personal qualities of an individual in a social or work } \\
\text { setting. }\end{array}$ \\
\hline & Optimism & The confidence that things will happen for the best or that desired goals will be attained. \\
\hline & Intentions & A conscious decision to perform a behaviour or a resolve to act in a certain way. \\
\hline & Goals & Mental representations of outcomes or end states that an individual wants to achieve. \\
\hline & Beliefs about consequences & $\begin{array}{l}\text { Acceptance of the truth, reality or validity about an ability, talent or facility that a person can put to } \\
\text { constructive use. }\end{array}$ \\
\hline & Reinforcement & $\begin{array}{l}\text { Increasing the probability of a response by arranging a dependent relationship, or contingency, } \\
\text { between the response and a given stimulus. }\end{array}$ \\
\hline & Emotion & $\begin{array}{l}\text { A complex reaction pattern, involving experiential, behavioural, and physiological elements, by which } \\
\text { the individual attempts to deal with a personally significant matter or event. }\end{array}$ \\
\hline
\end{tabular}


'deeper' understanding of healthcare workers' perceptions of barriers and enablers of hand hygiene compliance than is possible using quantitative methodologies. A qualitative paradigm using semi-structured interviews allows participants to describe their experiences and issues in their own language, and gives them the flexibility to describe the unique insights of their professional group, which may not be obvious to a researcher outside that group [18].

Based upon the analysis of these interviews, suggestions for the types of interventions that may be effective in improving the $\mathrm{HH}$ compliance of healthcare providers in ICU will be briefly identified. In this way, our intention is to present a novel, user-friendly method, grounded in behavioural change theory, that will allow infection control and quality improvement teams to explore, diagnose and understand the barriers and enablers to $\mathrm{HH}$ compliance in their own units and hospitals, and thereby prescribe appropriate interventions to address their local challenges in a targeted way.

\section{Method}

\section{Research design}

This qualitative study used semi-structured interviews with ICU doctors and nurses. The study has been reported in accordance with the Standards for Reporting Qualitative Research (SRQR) [19].

\section{Setting}

The participants were healthcare providers from two ICUs in hospitals in the Republic of Ireland. The ICUs had 25 and 5 beds respectively.

\section{Ethical considerations}

Ethical approval was obtained from the ethics boards of both participating hospitals. All participants provided written informed consent prior to the interview.

\section{Participants}

A total of 26 participants (12 ICU nurses, 11 anaesthetic specialist registrars, and three anaesthetic senior house officers) completed interviews as part of this study. There were no individuals approached during recruitment who declined to participate. Sixteen participants were female, 10 were male.

\section{Interview schedule}

The semi-structured interview schedule (see Additional file 1) was developed based upon the COM-B model. Accordingly, questions were developed relating to participants' capability, opportunity, and motivation to engage in $\mathrm{HH}$ behaviour. The capability questions asked participants about the training they had received and about their confidence in their knowledge of $\mathrm{HH}$ protocols.
The opportunity questions were designed to assess whether sufficient resources were available to make $\mathrm{HH}$ compliance possible (e.g. time, facilities), and whether the social environment supported $\mathrm{HH}$ behaviour (e.g. other healthcare providers engage in $\mathrm{HH}$ ). The motivation questions were concerned with assessing the respondents' belief in the utility of $\mathrm{HH}$, and whether they, and others in the unit, strive for high levels of appropriate $\mathrm{HH}$ behaviour.

The COM-B model was used to develop the interview guide, rather than the TDF, which was later used as a coding framework for the analysis. Following discussion with ICU staff as part of the development of the interview protocol, it was believed that the TDF was too constrictive to use to develop the interview guide and may lead participants to provide specific provide opinions about the topic that fit into the framework. That is, asking questions about barriers and enablers more broadly, under the COM-B headings, would allow comments about environmental resources, social support, and other factors to arise naturally, rather than being "prompted" by more narrow questions about individual domains of the TDF. This had the added advantage of keeping the interview schedule relatively short, as it did not have to cover all the domains of the TDF individually. The more comprehensive and granular TDF was later used as a coding framework for analysis. The interview protocol was piloted with two nurses and a doctor and found to be acceptable.

\section{Procedure}

The interviews were carried out by three of the authors (one psychologist and two medical doctors) between February 2017 and November 2017. Participants were recruited using purposive sampling and snowball techniques. The psychologist did not know the participants that she interviewed. However, the two doctors did know the participants that they interviewed. Interviews were conducted at a place and time convenient to the interviewee. Interviews were conducted in person, audio recorded and transcribed. Audio recordings of the interviews were destroyed after transcription. Interviews were conducted on-site. The median duration of the interviews was $4 \mathrm{~min} 41 \mathrm{~s}$. A stopping criterion of two interviews was used for doctors and nurses. The stopping criterion refers to the number of interviews to be conducted with in each sample group without new themes emerging. The sample frame included nurses and doctors who worked in ICU.

\section{Analysis}

To ensure rigour and trustworthiness of the data, content analysis was performed by two researchers, both psychologists who had not been involved in recruitment 
for, or conducting of, the interviews. No software was used to support the analysis. The researchers annotated printed copies of each interview as they worked through the content analysis process. A deductive content analysis approach was used to analyse the interview data, in which the analysis was planned and operationalised based on existing knowledge with the goal of testing a given theory [20]. The TDF was used as the initial framework for classifying the interview data. The reason for using the TDF, as opposed to the COM-B domains, was due to the extra granularity in coding provided by the TDF domains. After having read and coded the interviews independently, the two coders compared their codes and resolved disagreements through discussion to achieve consensus. Following completion of the analysis, exemplar quotes for each of the domains were chosen by consensus between the two researchers. The number of interviewees that made comments related to each of the 14 TDF domains was also recorded.

\section{Results}

The number and percentage of participants who made comments relating to each of the 14 TDF domains is provided in Table 2. It was found that the TDF was appropriate for analysing the data and all of the comments made by the participants could be classified using the TDF. A discussion of each of the TDF domains with exemplar comments made by the participants are provided below under the associated dimensions of the capability, opportunity and motivation dimensions of the COM-B model.

\section{Capability dimension \\ Knowledge}

The majority of participants $(n=22,84.6 \%)$ confirmed knowledge of the reasons for, and importance of, $\mathrm{HH}$ compliance. For example, one participant noted that "hand hygiene protocols are important in health care industry just for prevention of cross-infection" (P3, Doctor).

\section{Skill}

All but one $(n=25,96.2 \%)$ all of the interviewees described training in hand hygiene and were satisfied with the $\mathrm{HH}$ compliance training they had received. Several ( $n=15,57.7 \%)$ also described the necessity for ongoing top-up training. It was expressed by one of the doctors that "in this hospital there was about two structured training sessions with one infection control (person) and the second one with nursing staff" (P20, Doctor).

\section{Beliefs about capabilities}

Some of the doctors and nurses interviewed expressed different levels of confidence in their ability to carry out appropriate $\mathrm{HH}$ behaviours. Confidence may be higher among physicians, who were more likely to express confidence in their own $\mathrm{HH}$ knowledge and skills as compared to nurses. To illustrate, a doctor stated that "I' $m$ confident in my knowledge of my hand hygiene protocols and I don't feel I need a lot more hand hygiene protocols to be taught to me" (P4, Doctor), whereas a nurse said, "you can never have enough. It's always good to be reminded and refreshed" (P15, Nurse).

Table 2 Number and percentage of comments made by participants $(n=26)$ corresponding with each TDF domain

\begin{tabular}{ll}
\hline TDF Domain & No of participants who mentioned domains (\%) $(\mathrm{n}=26)$ \\
\hline Capability dimension & $22(84.6 \%)$ \\
- Knowledge & $25(96.2 \%)$ \\
- Skills & $16(61.5 \%)$ \\
- Beliefs about capabilities & $3(11.5 \%)$ \\
- Behavioural regulation & $11(42.3 \%)$ \\
- Memory, attention, and decision processes & \\
Opportunity dimension & $21(80.8 \%)$ \\
- Social influences & $26(100 \%)$ \\
- Environmental context and resources & \\
Motivation dimension & $14(53.9 \%)$ \\
- Social/professional role \& identity & 0 \\
- Optimism & 0 \\
- Intentions & $4(15.4 \%)$ \\
- Goals & $19(73.1 \%)$ \\
- Beliefs about consequences & $12(46.2 \%)$ \\
- Reinforcement & $7(26.9 \%)$ \\
- Emotion &
\end{tabular}




\section{Behavioural regulation}

This category was used infrequently (see Table 2). Only three participants (11.5\%) reported checking their own behaviour or consciously reminding themselves to perform $\mathrm{HH}$. To illustrate, one of the nurses said, "once you have this patient stable, then you have to look back and say what did we do well here? ... So you're always reviewing what you've done in the context of could I have caused an infection to this patient?" (P25, Nurse).

\section{Memory, attention, and decision processes}

Nurses in particular were more likely to describe $\mathrm{HH}$ as a habitual behaviour ( $n=5$ nurses $(41 / 7 \%), n=1$ doctor (7.1\%)). This point is illustrated by the following quote: "you just do it unknown to yourself" (P12, Nurse). However, $11(42.3 \%)$ of the participants recognised that they can sometimes forget to perform HH behaviour. For example, one of the nurses interviewed stated that "you might get so busy and engrossed in your work that you might forget" (P15, Nurse).

\section{Opportunity dimension Social influences}

Nurses were more likely than doctors to describe staff expressly encouraging and supporting one another in their HH compliance ( $n=7$ nurses (58.3\%), $n=2$ doctors (14.3\%)). To illustrate, "it's just a constant reminder, the CNMs [Clinical Nurse Managers] would be constantly prompting you" (P12, Nurse). Two nurses (16.7\%) also mentioned intergroup conflicts, whereby a doctor or more senior member of staff reacted badly to their behaviour being corrected by a nurse. For example, "I as a nurse would say to a doctor or a senior consultant, 'please put on that', they don't like being told" (P25, Nurse).

Doctors more frequently referred to observed social norms as an influence on their compliance as compared to nurses ( $n=3$ doctors (21.4\%), $n=1$ nurse (8.3\%). An example quote of this type is, "it's from the top down. All the consultants wash their hands, the nurses all wash their hands and everybody else washes their hands ... so there's a culture for it." (P2, Doctor).

\section{Environmental context and resource}

Nurses in particular reported receiving direct reminders and encouragement from colleagues to engage in $\mathrm{HH}$. By way of illustration, "you remind each other. When you're at a bedside and you go, "Will you wash your hands there before you come in?' ... you'd forget sometimes, you know?" (P15, Nurse).

Twelve (46.2\%) participants described a climate in which members of staff are aware of and observe one another's $\mathrm{HH}$ behaviour; participants said that this motivates compliance. One of the nurses noted that "every single nurse will give out to you if you don't .... They'll tell you ... that you need to (P13, Nurse). Similarly, one of the doctors stated that "there's nurses constantly hassling you to do it [hand hygiene]" (P5, Doctor).

Nurses also often described situations where material resources were lacking ( $n=6$ nurses, 50\%). For example, "in a seven bedded ward or a twelve bedded ward, you wouldn't have sinks at every bedside." (P12, Nurse). Although not mentioned by doctors, nurses also described staffing levels as an occasional impediment to compliance ( $n=8$ nurses, 66.7\%). For example, "short staffed on the unit ... when people go on lunch, then there's less people around and then things can just crack off and that can delay you." (P18, Nurse).

\section{Motivation dimension}

Social/professional role and identity. Protecting patients through $\mathrm{HH}$ was recognised as part of the job. For example, "this $[\mathrm{HH}]$ is an important part of managing your introduction to patients. It's part of being a good doctor" (P2, Doctor). However, it was also pointed out that nurses were less likely to miss $\mathrm{HH}$ opportunities as compared to doctors. To illustrate, "I've noticed the nurses are quite good. Doctors, you have to prompt them a few times" (P13, Nurse).

\section{Optimism and intentions}

There were no comments related to these two domains of the TDF in any of the interviews.

\section{Goals}

A small number of participants $(n=4 ; 15.4 \%)$ commented that the $\mathrm{HH}$ goal of $90 \%$ was unachievable. One nurse said, "I do believe that if you were to do it exactly, it would be impossible, you know?" (P18, Nurse), while a registrar described the task of perfect compliance as " $a b$ solutely impossible ... not achievable ... not practical" and said that "if you were to follow every movement of hand hygiene you'd get no work done ... you'd end up with some kind of contact dermatitis from ... washing your hands forty times a day" (P5, Doctor).

\section{Beliefs about consequences}

A majority of participants $(n=17 ; 65.4 \%)$ expressed the belief that poor $\mathrm{HH}$ contributes to infection and poor patient outcomes: "The lack of action could harm somebody" (P9, Doctor). However, there was also a recognition by eight (23.1\%) of the participants that $\mathrm{HH}$ measures "can be very hard on your hands. You'd feel like ... your skin would be breaking and everything" (P12, Nurse).

\section{Reinforcement}

Nine (34.6\%) of the participants commented that healthcare providers are motivated to perform $\mathrm{HH}$ by the 
demonstrated or evidenced protection it offers to themselves, as well as their patients. "As someone who works in ICU, I don't want to get colonised with resistant bacteria either" (P27, Doctor). Five (19.2\%) of the interviewees pointed out that positive feedback from audits and infection transmission data encourages continued compliance with $\mathrm{HH}$ : "If you're looking up at the board ... and you think we've been free of MRSA [Methicillin-resistant Staphylococcus aureus] as acquired on the units for ... that's brilliant that you see that we're doing something right in that sense." P18, Nurse).

\section{Emotion}

Six (23.1\%) participants referred to ethical principles that encourage them to engage in hand hygiene. Commitment to caring for the patient ("in your head you're thinking, you know, first do no harm" (P13, Nurse)) and reciprocity or the 'golden rule' ("if I would apply that rule to myself, then I definitely should apply it to others" (P27, Doctor)) both support good hand hygiene practices.

\section{Discussion}

Substantial resources are invested in $\mathrm{HH}$ improvement efforts in ICUs [4], with considerable efforts focused upon observations of $\mathrm{HH}$ compliance. However, it is important that these limited resources are used effectively [21]. Although direct observation is considered the 'gold standard' method of measuring $\mathrm{HH}$ compliance [22], it only supports a very limited understanding of the reasons why a healthcare professional does, or does not, engage in appropriate $\mathrm{HH}$ behaviours. Therefore, there is a need to consider other complementary methods to develop an understanding of the determinants of current and desired behaviours that support $\mathrm{HH}$ compliance, and thereby use this understanding to develop theoretically-based and tailored interventions. The purpose of the study reported in this paper was to apply a commonly used behavioural change theory to gain an understanding of the barriers and enablers $\mathrm{HH}$ behaviours, and to use this information to consider the types of interventions that may be effective in improving the $\mathrm{HH}$ compliance of ICU staff. The discussion will consider the findings within the capabilities, opportunities, and motivation dimensions of the COM-B model and their associated TDF domains.

\section{Capabilities dimension}

It was clear from the ICU staff interviews that the participants were confident in their capabilities to carry out appropriate $\mathrm{HH}$ behaviours. The vast majority of participants reported having the necessary knowledge, skills, and capabilities to perform appropriate $\mathrm{HH}$ behaviours. The fact that healthcare workers believe that they have the requisite knowledge and skills to carry out $\mathrm{HH}$ has also been found in other studies [23]. However, observations of 335 moments of $\mathrm{HH}$ in the two ICUs at which the interviewees worked found an overall compliance of $64 \%$ [24]. This lack of concordance between selfreported and observed $\mathrm{HH}$ compliance has also been reported elsewhere [25]. Therefore, although training may be required, interventions designed to increase $\mathrm{HH}$ knowledge or skills may not impact $\mathrm{HH}$ compliance, as ICU staff believe they have the requisite knowledge and skill to carry out $\mathrm{HH}$ when appropriate.

This perceived overestimation of $\mathrm{HH}$ compliance is an important barrier to engagement with training and educational interventions, and it impacts all of the TDF domains within the capability dimension of the COM-B model. It is suggested that this overestimation may partially be attributed to the fact that ICU staff generally only receive information on $\mathrm{HH}$ compliance at a unit level, and do not receive feedback on their own individual $\mathrm{HH}$ compliance. It is suggested that there is a need for targeted $\mathrm{HH}$ training in which ICU staff receive direct and individualised feedback on actual performance at the bedside (addresses the TDF domains of knowledge and beliefs about capabilities; see Table 1), and are provided guidance on how to address deficiencies in $\mathrm{HH}$ compliance at the time at which the $\mathrm{HH}$ behaviour was performed (addresses the TDF domains of knowledge, skills, behavioural regulation, memory, attention, and decision processes; see Table 1). Sustained individualised feedback is supported by a number of empirical studies, indicating that it improves adherence to guidelines and that it is welcomed by healthcare workers [17, 26, 27].

The influence of professional group is also important. While the doctors in our study almost universally reported confidence in their $\mathrm{HH}$ performance, recent research and review work indicates that physicians are often excluded from studies of $\mathrm{HH}$ compliance and they tend to underperform in $\mathrm{HH}$ compliance compared to their colleagues in nursing and allied health professions [27-30]. Professional groups respond differently to interventions [27], and so targeted profession-specific training, taking account of their particular perspectives and challenges, is an important avenue for future research to explore.

\section{Opportunity dimension}

Social influence was regarded as being important in encouraging $\mathrm{HH}$ compliance by the interviewees, particularly by nurses. The sense of being watched or monitored, and explicit encouragement and reminders from peers were all identified as reinforcing or motivating factors in promoting $\mathrm{HH}$ compliance. In a study of the attitudes to $\mathrm{HH}$ of Canadian doctors using the TDF, the social environment and role models were also found to be important [3]. However, although persuasion (use of communication to produce positive or negative feelings that may promote engagement in a behaviour) is a 
commonly used $\mathrm{HH}$ compliance intervention in the ICU, a recent systematic review found no examples of the use of modelling (highlighting examples of desired behaviour in order to encourage others to emulate this behaviours) to improve $\mathrm{HH}$ compliance [4]. Encouraging leaders to act as 'champions' for $\mathrm{HH}$ may yield significant benefits [31]. Modelling of appropriate $\mathrm{HH}$ behaviours by senior leaders may be particularly important among doctors given the hierarchy and the apprenticeship model of training. A modelling-focused intervention would address both the social influences and environmental context and resources TDF domains.

The results presented here indicate some degree of mutual support between members of staff for hand hygiene; staff are reminded and encouraged to perform hand hygiene, both within and across professional groups. Research suggests that this 'speaking up' culture, led in our sample by women, is worth encouraging; although staff often fear speaking up due to concerns about reprisal, the perceived safety risk, and the appropriateness and perceived efficacy of speaking up [32, 33], initial efforts to speak up frequently can help hand hygiene to become part of the social norm on a ward, leading to less need to speak up over time [34].

Nurses in particular also commented on the impact of low staffing levels on $\mathrm{HH}$ compliance. $\mathrm{HH}$ compliance is a time-consuming activity that does have the potential to negatively impact patient safety if there is a shortage of staff. In an evaluation of how long nursing staff must spend in order to be $100 \%$ compliant with $\mathrm{HH}$ recommendations, it was found that 12 nurses working in an eight bed ICU would devote a total of 4 hours to $\mathrm{HH}$ if using alcohol hand gel, or 16 hours if using soap and water across an eight-hour shift [35]. Therefore, levels of staffing are a factor that must be considered if $\mathrm{HH}$ compliance is to be improved.

\section{Motivation dimension}

The interviewees were motivated to carry out $\mathrm{HH}$ behaviours, and it was recognised that $\mathrm{HH}$ is an important part of their job and crucial in preventing infection. The desire to perform $\mathrm{HH}$ to protect themselves was mentioned by a third of the interviewees in our study. The propensity towards prioritising the protection of oneself from infection has also been found in other studies of $\mathrm{HH}$ compliance [28, 36, 37], including observations carried out in the two ICUs from which the interviewees in our study worked [24]. In the observations carried out in the ICUs, the staff were 55\% compliant before an aseptic technique, as compared to $89 \%$ compliant after patient contact [24]. Therefore, interventions focused on those $\mathrm{HH}$ moments that protect the patient are merited. It is suggested that the direct observation and feedback intervention recommended above under the capabilities domain could also be an effective intervention for bringing about a focus on the $\mathrm{HH}$ moments that protect the patient and address the intentions, goals, and reinforcement domains of the TDF.

The Optimism and Intention domains of the TDF were not used to classify and of the participants' comment. That all domains of the TDF were not used to describe the interview data was expected. Squires et al. [3] conducted TDF domain interviews with doctors about hand hygiene compliance and found that four TDF domains were not relevant to physician hand hygiene compliance (Optimism, Intention, Reinforcement, and Emotion). The TDF is a broad overarching approach to understanding many behaviours in a range of settings; it is unsurprising, therefore, that not all domains were relevant in this study's specific setting.

\section{Limitations}

The study had a number of limitations. First, only doctors and nurses were recruited using a convenience sampling approach. $\mathrm{HH}$ is also crucial for other members of staff, including healthcare assistants, therapists, porters, and catering staff, and it important to capture their insights also to ensure the efficacy of any intervention that follows. Second, as is common with all qualitative research, the generalisability of the findings may be questioned. Nevertheless, there is some support for the generalisability of the findings as they are in broad agreement with other studies of $\mathrm{HH}$ in hospital settings $[2,3,38]$. Third, the respondents may have felt some pressure to provide socially desirable responses to some of the questions. We hope that this effect was limited by the fact that the interviews were conducted by researchers from outside the hospital and the responses were anonymised. Finally, this paper only reports one method of exploring attitudes to $\mathrm{HH}$ compliance. This is certainly a limitation in terms of obtaining a broad understanding of $\mathrm{HH}$ behaviour, particularly when considering the discordance with the $\mathrm{HH}$ observation data. This is the very reason we recognise the importance of taking a multi-methods approach to understanding $\mathrm{HH}$ behaviour. In fact, these interviews were conducted as part of a larger project exploring $\mathrm{HH}$ compliance in Irish ICUs in which other methods of assessing $\mathrm{HH}$ behaviour were also utilised [4, 24, 29, 39].

\section{Conclusions}

Best practice for improving hand hygiene in ICUs remains unestablished [4], and compliance is less than optimal [29]. Although direct observation is considered the 'gold standard' method of measuring $\mathrm{HH}$ compliance [22], it provides little understanding as to why a particular behaviour did or did not occur. This study has demonstrated that short interviews with ICU staff, founded 
on appropriate behavioural change theory, can provide a depth of understanding into $\mathrm{HH}$ behaviour that can complement, and inform, data collected from other methods (e.g. direct observation). This understanding can then be applied to the design of theoretically valid interventions that are appropriately tailored to the needs of a specific unit, and which will have an increased likelihood of success. The work presented here offers a novel, user-friendly method for infection control and quality improvement teams to explore, diagnose and understand the factors influencing $\mathrm{HH}$ compliance in their own units and hospitals, thereby offering an opportunity to address their specific challenges in a targeted way. This study forms part of a larger project, one aim of which is to develop a 'toolkit' of potential interventions that may be applied by local teams, specified according to the findings of an investigation like the one presented here. Although a 'one size fits all' approach is desirable from the perspective of convenience, there is a risk that, in fact, 'one size fits nobody', and improvement efforts that fail to acknowledge local circumstances and challenges constitute wasted opportunities to bring about meaningful long-term change.

\section{Supplementary information}

Supplementary information accompanies this paper at https://doi.org/10. 1186/s12913-020-05215-4.

Additional file 1. Semi-structured interview schedule. Description of data: Contains the schedule of questions used in the semi-structured interviews.

\section{Abbreviations}

CNM: Clinical Nurse Manager; COM-B model: Capability Opportunity Motivation and Behaviour model; $\mathrm{HH}$ : Hand hygiene; ICU: Intensive Care Unit; SRQR: Standards for Reporting Qualitative Research; TDF: Theoretical Domains Framework

\section{Acknowledgements}

The authors wish to acknowledge the support of the hospital and participants in completing this study.

\section{Authors' contributions}

POC, SL, JM, and MB were involved in the design and planning of the study. $\mathrm{SL}, \mathrm{RB}, \mathrm{RM}$, and OT made a substantial contribution to recruitment and data collection for the study. $\mathrm{AH}$ and $\mathrm{CM}$ completed the transcription of the interviews. POC, SL, AH, CM, and $\mathrm{KL}$ contributed to the analysis of the data. $\mathrm{KL}, \mathrm{POC}$, and SL drafted the initial manuscript. All authors contributed to the redrafting of the manuscript, approved the submitted version of the manuscript, and have agreed to be personally accountable for their contribution and attest to the accuracy or integrity of the subm. All authors reviewed and approved the final manuscript prior to submission.

\section{Funding}

Funding was obtained from the Irish Health Research Board. The funders did not input on study design, data collection, data analysis, interpretation, or approve the final manuscript.

\section{Availability of data and materials}

The datasets used and/or analysed in the current study are available from the corresponding author on reasonable request.

\section{Ethics approval and consent to participate}

Ethical approval was obtained from the ethics committees of Galway University Hospital and Sligo University Hospital. Written informed consent was obtained from all participants included in the study.

\section{Consent for publication}

Not applicable.

\section{Competing interests}

Authors Paul O'Connor and Sinéad Lydon are Associate Editors of this journal.

\section{Author details}

${ }^{1}$ Discipline of General Practice, School of Medicine, National University of Ireland Galway, Co. Galway, Ireland. ${ }^{2}$ Irish Centre for Applied Patient Safety and Simulation, School of Medicine, National University of Ireland Galway, Co. Galway, Ireland. ${ }^{3}$ School of Medicine, National University of Ireland Galway, Co. Galway, Ireland. ${ }^{4}$ School of Psychology, National University of Ireland Galway, Co. Galway, Ireland. ${ }^{5}$ Department of Anaesthesia \& Intensive Care, University Hospital Galway, Co. Galway, Ireland. ${ }^{6}$ The College of Anaesthesiologists of Ireland, Dublin, Ireland. ${ }^{7}$ Department of Anaesthetics, Sligo University Hospital, Sligo, Ireland.

Received: 13 February 2020 Accepted: 13 April 2020

Published online: 25 April 2020

\section{References}

1. Maskerine CLM. Improving adherence to hand hygiene among health care workers. J Contin Educ Health Prof. 2006;26(3):244-51.

2. Dyson J, Lawton R, Jackson C, Cheater F. Development of a theory-based instrument to identify barriers and levers to best hand hygiene practice among healthcare practitioners. IS. 2013;8:111.

3. Squires JE, Linklater S, Grimshaw JM, Graham ID, Sullivan K, Bruce N, et al. Understanding practice: factors that influence physician hand hygiene compliance. Infect Control Hosp Epidemiol. 2014;35(12):1511-20.

4. Lydon S, Power M, McSharry J, Byrne M, Madden C, Squires JE, et al. Interventions to improve hand hygiene compliance in the ICU: a systematic review. Crit Care Med. 2017;45(11):e1165-e72.

5. Gould DJ, Moralejo D, Drey N, Chudleigh JH, Taljaard M. Interventions to improve hand hygiene compliance in patient care. Cochrane Database Syst Rev. 2017:9:CD005186.

6. Bonetti D, Eccles M, Johnston M, Steen N, Grimshaw J, Baker R, et al. Guiding the design and selection of interventions to influence the implementation of evidence-based practice: an experimental simulation of a complex intervention trial. Soc Sci Med. 2005;60(9):2135-47.

7. Davies P, Walker AE, Grimshaw JM. A systematic review of the use of theory in the design of guideline dissemination and implementation strategies and interpretation of the results of rigorous evaluations. IS. 2010:5:14.

8. Grol RP, Bosch MC, Hulscher ME, Eccles MP, Wensing M. Planning and studying improvement in patient care: the use of theoretical perspectives. Milbank Q. 2007;85(1):93-138.

9. Srigley JA, Corace K, Hargadon DP, Yu D, MacDonald T, Fabrigar L, et al. Applying psychological frameworks of behaviour change to improve healthcare worker hand hygiene: a systematic review. J Hosp Infect. 2015; 91(3):202-10

10. Michie $S$, Atkins L, West R. The behaviour change wheel: a guide to developing interventions. London: Silverback Publishing; 2014.

11. Michie S, van Stralen MM, West R. The behaviour change wheel: a new method for characterising and designing behaviour change interventions. Implement Sci. 2011;6:42.

12. Cane J, O'Connor D, Michie S. Validation of the theoretical domains framework for use in behaviour change and implementation research Implement Sci. 2012;7:37.

13. Atkins L, Francis J, Islam R, O'Connor D, Patey A, Ivers N, et al. A guide to using the theoretical domains framework of behaviour change to investigate implementation problems. Implement Sci. 2017;12(1):77.

14. Dyson J, Lawton R, Jackson C, Cheater F. Does the use of a theoretical approach tell us more about hand hygiene behaviour? The barriers and levers to hand hygiene. J Infect Prev. 2011;12(1):17-24.

15. Fuller C, Besser S, Savage J, McAteer J, Stone S, Michie S. Application of a theoretical framework for behavior change to hospital workers' real-time 
explanations for noncompliance with hand hygiene guidelines. Am J Infect Control. 2014;42(2):106-10.

16. Smith JD, Corace KM, MacDonald TK, Fabrigar LR, Saedi A, Chaplin A, et al. Application of the theoretical domains framework to identify factors that influence hand hygiene compliance in long-term care. J Hosp Infect. 2019; 101(4):393-8.

17. Smiddy MP, Murphy OM, Savage E, Browne JP. The influence of observational hand hygiene auditing on consultant doctors' hand hygiene behaviors: a qualitative study. Am J Infect Control. 2019;47(7): 798-803 e1.

18. Smiddy MP, OC R, Creedon SA. Systematic qualitative literature review of health care workers' compliance with hand hygiene guidelines. Am J Infect Control. 2015:43(3):269-74.

19. O'Brien BC, Harris IB, Beckman TJ, Reed DA, Cook DA. Standards for reporting qualitative research: a synthesis of recommendations. Acad Med. 2014;89(9):1245-51.

20. Elo S, Kyngas H. The qualitative content analysis process. J Adv Nurs. 2008; 62(1):107-15.

21. Thu LTA, Thoa VTH, Van Trang DT, Tien NP, Van DT, Anh LTK, et al. Costeffectiveness of a hand hygiene program on health care-associated infections in intensive care patients at a tertiary care hospital in Vietnam. Am J Infect Control. 2015;43(12):e93-9.

22. Boyce J, Chartier Y, Chraiti M, Cookson B, Damani N, Dharan S. WHO guidelines on hand hygiene in health care. Geneva; 2009.

23. Lee SS, Park SJ, Chung MJ, Lee JH, Kang HJ, Lee JA, et al. Improved hand hygiene compliance is associated with the change of perception toward hand hygiene among medical personnel. Infect Chemother. 2014;46(3):165-71.

24. Lydon S, Greally C, Tujjar O, Reddy K, Lambe K, Madden C, et al. Psychometric evaluation of a measure of factors influencing hand hygiene behaviour to inform intervention. J Hosp Infect. 2019.

25. Jenner EA, Fletcher BC, Watson P, Jones FA, Miller L, GM S. Discrepancy between self-reported and observed hand hygiene behaviour in healthcare professionals. J Hosp Infect. 2006;1 (63):418-22.

26. Hysong SJ, Best RG, Pugh JA. Audit and feedback and clinical practice guideline adherence: making feedback actionable. Implement Sci. 2006;1:9.

27. Hoffmann M, Sendlhofer G, Gombotz V, Pregartner G, Zierler R, Schwarz C, et al. Hand hygiene compliance in intensive care units: An observational study. Int J Nurs Pract. 2019:e12789.

28. Allegranzi B, Gayet-Ageron A, Damani N, Bengaly L, McLaws M-L, Moro M-L, et al. Global implementation of WHO's multimodal strategy for improvement of hand hygiene: a quasi-experimental study. Lancet Infect Dis. 2013;13(10):843-51.

29. Lambe KA, Lydon S, Madden C, Vellinga A, Hehir A, Walsh M, et al. Hand hygiene compliance in the intensive care unit: a systematic review. Crit Care Med. 2019.

30. Sharma R, Sharma M, Koushal V. Hand washing compliance among healthcare staff in Intensive Care Unit (ICU) of a multispecialty hospital of North India. Journal of Hospital Administration. 2012;1(2).

31. Sakihama T, Honda H, Saint S, Fowler KE, Kamiya T, Sato Y, et al. Improving healthcare worker hand hygiene adherence before patient contact: a multimodal intervention of hand hygiene practice in three Japanese tertiary care centers. J Hosp Med. 2016:11(3):199-205.

32. Lyndon A, Sexton JB, Simpson KR, Rosenstein A, Lee KA, Wachter RM. Predictors of likelihood of speaking up about safety concerns in labour and delivery. BMJ Qual Saf. 2012;21(9):791-9.

33. Okuyama A, Wagner C, Bijnen B. Speaking up for patient safety by hospitalbased health care professionals: a literature review. BMC Health Services Research. 2014;14(61).

34. Linam WM, Honeycutt MD, Gilliam CH, Wisdom CM, Deshpande JK. Impact of a successful speaking up program on health-care worker hand hygiene behavior. Pediatr Qual Saf. 2017;2(4):e035.

35. Voss A, AF W. No time for handwashing!? Handwashing versus alcoholic rub can we afford 100\% compliance? Infect Control Hosp Epidemiol. 1997;18(3): 205-8.

36. Kolola T, Gezahegn T. A twenty-four-hour observational study of hand hygiene compliance among health-care workers in Debre Berhan referral hospital. Ethiopia Antimicrob Resist Infect Control. 2017;6:109.

37. Wetzker W, Bunte-Schonberger K, Walter J, Pilarski G, Gastmeier P, Reichardt C. Compliance with hand hygiene: reference data from the national hand hygiene campaign in Germany. J Hosp Infect. 2016;92(4):328-31.
38. Madden C, Lydon S, Walsh C, O'Dowd E, Fox S, Lambe K, Tujjar O, Greally C, Power M, Bates J, O'Connor P. What are the predictors of hand hygiene compliance in the Intensive Care Unit? A cross-sectional observational study. (in preparation).

39. Madden C, Lydon S, Lambe K, O'Connor P. Irish policy-makers' perceptions of barriers and facilitators to hand hygiene compliance. Ir Med J. 2019; 112(4):914.

\section{Publisher's Note}

Springer Nature remains neutral with regard to jurisdictional claims in published maps and institutional affiliations.
Ready to submit your research? Choose BMC and benefit from:

- fast, convenient online submission

- thorough peer review by experienced researchers in your field

- rapid publication on acceptance

- support for research data, including large and complex data types

- gold Open Access which fosters wider collaboration and increased citations

- maximum visibility for your research: over $100 \mathrm{M}$ website views per year

At BMC, research is always in progress.

Learn more biomedcentral.com/submissions 